\title{
Solving Vibrating Spring Problem Using Variational Formulation
}

\author{
Ameera N. Alkiffai ${ }^{a}$, Zainab M. Reda ${ }^{\text {b }}$ \\ ${ }^{\text {a,b }}$ College of Education for Girls, Kufa University, Iraq.
}

Article History: Received: 11 January 2021; Accepted: 27 February 2021; Published online: 5 April 2021

\begin{abstract}
This scientific study is concerned with an integral equation, especially with Volterra integral equation and used new variational formulation which corresponds on the linear operator $\mathrm{L} u=f$. The vibrating spring equation has been solved using this approach.
\end{abstract}

Keywords: Integral Equation, Variational Formulation, Vibrating Spring Equation.

\section{Introduction}

Integral equations appeared and used in various applied areas as important as differential equations but the integral formulations are more elegant and compact than the differential formulations due to the accuracy of solutions that obtained from the integral equations [3]. The present study works in solving Volterra integral equation directly using variational approach (Magri app) [1,9]. Moreover, a new approach will used for solving vibrating spring equation, this as an application to Magri approach. That physical phenomena of solids in free vibration is that the vibration of the solid induced by an instantaneous disturbance either in the form of a force or deformation of the supporting spring. The kinetic energy is stored in the movement of a mass where the measure of this movement is the velocity of the system and the continuous interchange between potential and kinetic energy is the vibration of the system $[4,7,13]$.

\section{Integral Equations}

An integral equation is an equation in which the unknown function $f(s)$ to be determined appears under the integral sign. A typical form of an integral equation in $f(s)$ is of the form,

$$
f(s)=\gamma(s)+\lambda \int_{v(s)}^{u(s)} k(s, t) f(t) d t(2.1)
$$

Where $k(s, t)$ is called the kernel of the integral equation, the function $\mathrm{\gamma}(s)$ is called the driving term, $u(s)$ and $v(s)$ are the limits of integration and may be both variables, constants or mixed. It is easily observed that the unknown function $f(s)$ appears under the integral sign as stated above, and out of the integral sign in most other cases. Integral equations arise naturally in physics, chemistry, biology and engineering applications modelled by initial value problems for a finite interval $[a, b]$. More details about the sources and origins of integral equations and as well how the initial value problem be converted to the form of integral equations can be found in [15]. Other problem whose direct representation in terms of differential equation and their auxiliary conditions may also be decreasing to integral equation [16].

Our goal is to classify integral equations, the kernel function in integral equations and to determine $f(s)$ that will satisfy (2.1). according to Magri approach.

\section{Types of Integral Equations}

According to the limits of integrations, the driving terms and the kernel, Arfken [4] gave a simple classification for integral equations, as follows:

1. If the limits of integration are fixed, the equation is called Fredholm integral equation, whereas if one of these limits is variable, then the equation will be Volterra integral equation.

2. If the unknown function appears only under the integral sing, we shall label it, first kind. While if it appears both inside and outside the integral sing, it will be labeled as, second kind. Symbolically, we write the Fredholm integral equation of the first kind and second kind respectively as follows:

$$
\gamma(s)=\lambda \int_{a}^{b} k\left(s, \mathrm{t}\left(f(t) d t, f(s)=\gamma(s)+\lambda \int_{a}^{b} k(s, \mathrm{t}) f(t) d t\right.\right.
$$

Whereas the Volterra integral equation of the first, second kind will be written respectively as: 


$$
\gamma(s)=\int_{a}^{s} k(s, t) f(t) d t, f(s)=\gamma(s)+\lambda \int_{a}^{s} k(s, t) f(t) d t \text { For more types, see [3, 2,4]. }
$$

\section{Classification of the Kernel of Integral Equations: [6]}

As mentioned earlier, the role of the known bivariate function $k(s, t)$ is quite significant both from the problem and its solution point-of-view. Mainly, we shall come across with the following classification of the kernel of the integral equations:

1. The kernel $k(s, t)$ is said to be symmetric (complex symmetric or Hermitian) if $k(s, t)=\bar{k}(t, s)$ (where the bar represents the complex conjugate). A real kernel is symmetric if $k(s, t)=k(t, s)$.

2. The kernel $k(s, t)$ is called separable or degenerate kernel which means that $k$ has been expressed as the sum of a finite number of terms, each of which is the product of function of $\mathrm{s}$ only and $\mathrm{t}$ only, $k(s, t)=\sum_{i=1}^{n} f_{i}(s) \gamma_{i}(t)$. Obviously, $f(\mathrm{~s})_{i}$ and $\gamma_{i}(t)$ are linearly independent (or else some of the terms will combine, and consequently, the number of terms will reduce). A degenerate kernel has a finite number of characteristic values.

3. The kernel of the form $k(s, t)=k(s-t)$ is called a difference kernel since it depends solely on the difference $(s-t)$.

\section{The Connection between Differential and Integral Equations: [5]}

There is a close connection between these kinds of equations many initial and boundary value problems can formulated as integral equations and vice versa. In general, the initial problems, dynamical systems and boundary value problem can be formulated as Volterra and Fredholm integral equations respectively.

Consider the differential problem (initial value problem):

$$
\grave{\mathrm{y}}(\mathrm{s})=f(s, \mathrm{y}), \mathrm{y}\left(s_{0}\right)=y_{0}
$$

then $\int_{s}^{s_{0}} y^{\prime}(t) d t=\int_{s_{0}}^{s} f(t, y(t)) d t$ which means that:

$$
\mathrm{y}(s)=y_{0}+\int_{s_{0}}^{s} f(t, y(t)) d t .
$$

On the other hand, if (2.3) holds, we get y $\left(s_{0}\right)=y_{0}$ and ỳ $(s=\mathrm{f}(s, y)$ which implies that (2.2) holds. Thus (2.2) and (2.3) are equivalent.

\section{The Variational Formulation}

Definition 1: (Linear Operator) [3]

A functional is an operator whose range lies on the real line $\mathrm{R}$ or in the complex $\mathcal{C}$. A functional $\beta$ on a linear space $U$ is called linear if for any $u_{1}, u_{2} \in \mathrm{U}$ and any scalar $\alpha$ we have:

1. $\beta\left(u_{1}+u_{2}\right)=\beta\left(u_{1}\right)+\beta\left(u_{2}\right)$.

2. $\beta\left(\alpha u_{1}\right)=\alpha \beta\left(u_{1}\right)$.

\section{Definition 2 (Bilinear Form) [12]:}

A functional $(u, v)$ depending on $u, v \in U$ is said to be bilinear form, if it satisfies the following properties for $w \in U$ and $\alpha \in R$ :

$(u+w, v)=(u, v)+\beta(w, v), \beta(\alpha u, v)=\alpha \beta(u, v)$ and

$\beta(u, v+w)=\beta(u, v)+\beta(u, v), \beta(u, \alpha v)=\alpha \beta(u, v)$.

Definition 3 (Non-Degenerate Bilinear Form) [1,11]:

The functional $(u, v)$ is denoted by $\langle u, v\rangle$ which called non- degenerate on the two linear space $U$ and $V$ if for every $u \in U$ and $v \in V$, the following conditions are hold:

1. $1 .<u, \bar{v} \geq 0$, then $\bar{v}=0$.

2. $\langle\bar{u}, v \geq 0$, hen $\bar{u}=0$.

Definition 4 (Symmetric Bilinear Form) [3]:

Let $\mathrm{U}$ and $\mathrm{V}$ be two normed linear spaces and $\langle u, v\rangle$ be a bilinear form, then $\mathrm{F}(u, v)=\langle u, v\rangle$ is said to be symmetric if: 
$\langle u, v\rangle=\langle v, u\rangle$, for all $\langle u, v\rangle \in \mathrm{U} \times \mathrm{V}$. It is clear that a linear operator $L$ is said to be symmetric linear operator with respect to chosen bilinear form $\langle u, v\rangle$, if:

$\langle\mathrm{L} u, v\rangle=\langle\mathrm{L} v, u\rangle, \forall m \in \mathrm{U}, n \in \mathrm{V}$.

$\langle u, v\rangle$ is saide onto be Symmetric.

For many examples of non-degenerates bilinear forms see [11].

\section{Theorem: [1]}

For a given symmetric linear operator $\mathrm{L}$ with respect to the chosen bilinear form $\langle u, v\rangle$, the solutions of the equation $\mathrm{L} u=f$ are the critical points of the functional:

Proof: see [3].

$$
\mathrm{F}[u]=<\mathrm{L} u, \mathrm{~L} u>-<f, u>
$$

\section{Approximated Solutions for Integral Equations}

Because of that some integral equations, particularly in non-homogeneous or non-linear cases, are not easy to analytically solve, an approximate method is needed. For solving Fredholm and Volterra integral equations, there are several normal approximate methods, see [3,8]. We addressed the use of variational formulation to solve Volterra integral equations in this paper.

\section{Example 1:}

Consider the following Volterra integral equation:

$$
\begin{gathered}
f(s)=s-\int_{0}^{s}(s-t) f(t) d t, \text { where its analytic solution is: } \\
f(s)=s-\frac{s^{3}}{3 !}+\frac{s^{5}}{5 !}=\sin s
\end{gathered}
$$

Using the linear operator $L=I+\int_{0}^{s}(s-t) f(t) d t$ and chosen bilinear form:

$$
\begin{gathered}
\left\langle u,>=\int_{s} u(s)(T-s) d s,\right. \text { to get the functional: } \\
{[f]=\frac{1}{2} \int_{0}^{s}\left[f(s)+\int_{0}^{s} k(s, t) f(t) d t\right]^{2} d s-\int_{0}^{s} \gamma(s)\left[f(s)+\int_{0}^{s} k(s, t) f(t) d t\right] d s^{v}(4.1)}
\end{gathered}
$$

The function $f(\mathrm{~s})$ can be written as a linear combination as:

and

$$
\begin{gathered}
f(\mathrm{~s})=a_{1} s+a_{2} s^{2}+a_{3} s^{3}, k(\mathrm{~s}, \mathrm{t})=(s-t) \text { and } \mathrm{\gamma}(\mathrm{s})=2 s \\
\text { So, } \int_{0}^{s} k(s, t) \gamma(t) d t=\frac{a_{1}}{2} s^{2}+\frac{a_{2}}{6} s^{3}+\frac{a_{3}}{12} s^{4}(4.2)
\end{gathered}
$$$$
\int_{0}^{1-s} k(s, t) y(t) d t=\left(\frac{a_{1}}{2}+\frac{a_{2}}{6}+\frac{a_{3}}{12}\right)+\left(-a_{1}+\frac{a_{2}}{2}+\frac{a_{3}}{3}\right) s+\left(\frac{a_{1}}{2}+\frac{a_{2}}{2}+\frac{a_{3}}{3}\right) s^{2}+\left(-\frac{a_{2}}{6}+\frac{a_{3}}{3}\right) s^{3}+\frac{a_{2}}{12} s^{4}
$$

substituting (4.3) and(4.2) in(4.1), to obtain $F[f]$. Now by equating the partial derivatives of the functional $F$ $[f]$ with respect to the coefficients of the solution $\left(a_{1}, a_{2}, a_{3}\right)$ to zero, we will get the critical points which give the linear system of equations for $a_{1}, a_{2}, a_{3}$ and by solving this system we have the values:

for more examples, see 2.4 in $[3,10]$.

$$
\begin{gathered}
\frac{83}{60} a_{1}+\frac{49}{72} a_{2}+\frac{383}{840} a_{3}=\frac{3}{4} \\
\frac{49}{72} a_{1}+\frac{509}{1260} a_{2}+\frac{169}{576} a_{3}=\frac{6}{15} \\
\frac{383}{840} a_{1}+\frac{169}{576} a_{2}+\frac{61122}{272160} a_{3}=\frac{10}{36} \\
a_{1}=1.2527, a_{2}=-4.5624, a_{3}=4.6541
\end{gathered}
$$

\section{Vibrating Spring Equation}


Phenomenon of vibration is the exchange of energy between potential (power) and kinetic (resuiting from motion) energy. Therefore, a vibrating system must have a component that stores potential energy. This component must also be capable of releasing the energy as kinetic energy. The simplest physical model for forced vibration is a simple mass-spring system subjected to an exciting force $\mathrm{F}(\mathrm{t})$ where $\mathrm{t}$ (time variable):

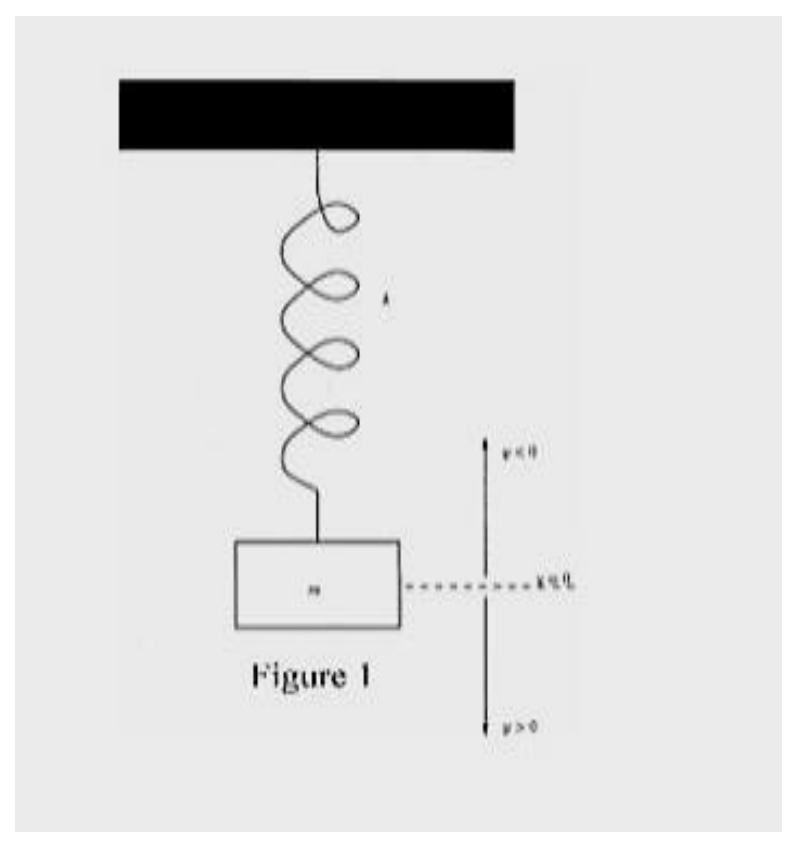

The differential equation for the instantaneous amplitudes of the vibrating mass under the influence of force $\mathrm{F}(\mathrm{t})$ becomes:

$$
m \frac{d^{2} y(t)}{d t^{2}}+A y(t)=\mathrm{F}(\mathrm{t})
$$

Equation (4.1) is a nonhomogeneous 2nd order differential equation As an application of variational formulation that is related to integral equation in physics is that the vibrating spring equation., for example, as follows[14] :

$$
f(s)=B s-\int_{0}^{s}(s-t) f(t) d t(4.5)
$$

Which is a second kind of Volterra integral equation. To solve this Volterra equation (4.5) using variational formulation, we have defined the linear operator as :

$$
\begin{aligned}
& L=I+\int_{0}^{s}(s-t) f(t) d t \text { with the bilinear form : } \\
& <u,>=\int_{s}^{s} u(s) v(t) d s \text {, the functional } F[f] \text { will be : } \\
& {[f]=\frac{1}{2}(L f, f)-(\mathbf{y}, \mathrm{f})} \\
& \qquad=\frac{1}{2} \int_{s}^{0}(I-I N) f(s)(I-I N) f(\mathrm{~s})-\mathrm{\gamma}(\mathrm{s})(I-I N) f(\mathrm{~s}) d \mathrm{~s} \\
& {[\mathrm{f}]=\frac{1}{2} \int_{0}^{s}\left[f(s)+\int_{0}^{s} k(s, t) f(t) d t\right]^{2} d s-\int_{0}^{s} \mathrm{\gamma}(\mathrm{s})\left[f(s)+\int_{0}^{s} k(s, t) f(t) d t\right] d s^{v}(4.6)}
\end{aligned}
$$

Suppose that the function $(t)$ as a linear combination of some basis as:

$f(t)=a_{1}+a_{1} \mathrm{~s}+a_{1} s^{2}$ As well as the $\mathrm{s}=0$ with the $\mathrm{B}=20$, by flowing the same procedure in Example (1), the critical points of the functional $F[f]$ in (4.5) are found by equating the derivatives of $F[f]$ with respect to the coefficient of the solution $\left(a_{1}, a_{2}\right.$ and $\left.a_{3}\right)$ to zero, then solve the obtained system of linear algebraic equations:

which will give the values of $a_{1}, a_{2}$ and $a_{3}$ :

which will give the values of $a_{1}, a_{2}$ and $a_{3}$ :

$$
\frac{83}{60} a_{1}+\frac{49}{72} a_{2}+\frac{383}{840} a_{3}=\frac{25}{2}
$$




$$
\begin{gathered}
\frac{49}{72} a_{1}+\frac{509}{1260} a_{2}+\frac{169}{576} a_{3}=\frac{22}{3} \\
\frac{383}{840} a_{1}+\frac{169}{576} a_{2}+\frac{10187}{45360} a_{3}=\frac{380}{72} \\
a_{1}=-0.1470, a_{2}=21.8210, a_{3}=-4.7089
\end{gathered}
$$

This result shortens a lot of efforts and time for programming rather than solving the required problem, since using the linear system is easier for programming, as differential equation where the solution is difficult. Moreover, using the linear system is easier for programming in order to solve it's physics equation vibrating spring equation.

\section{References}

1. AL Kiffai A.N; Noor N. Otaiwi, "Solve Storekeeper's Control Problem Using Variational Technique", Journal Dynamical and Control Systems, 2019.

2. AL kiffai A.N. "Solution of Inverse problem of Fredholm Integral Equation", Al - Qadisiyah Journal of Pure Science, vol: 9, No :3, P:114-118, 2004.

3. AL kiffai A.N. "Solution of Inverse Problem of Integral Equations Using Variational Technique" Ph. D Thesis, Babylon University, 2000.

4. Arfken G. "Mathematical Methods for Physicists", 3rd Edition, 1978.

5. Cannon J.R. "The One - Dimensional High Equation", Cambridge University Press, 2012.

6. D.C. Sharma; M.C. Goyal, "Integral Equations", PHI Learning Private Limited, Delhi, 2017.

7. Joseph A Macinante. "Seismic mountings for vibration isolation", Wiley, 1984.

8. Magri F. "Variational Formulation for Every Linear Equation", Int. Eng. Sci., 12, P:49 - 537, 1974.

9. Magri F. "Variational Formulation for Every Linear Equation", Int. Eng. Sci., 12, 537-547, 1974.

10. Noor N. Otaiwi, "Variational Technique For Solve Storekeeper's Control Problem as an Integral Equations", Ph.D Thesis, Kufa University, 2020.

11. Razdolsky L. Probability Based High Temperature Engineering: Creep and Structural Fire Resistance, Springer, 2017.

12. Reddy J.N. "Applied Functional Analysis and Variational Methods in Engineering", McGraw - Hill College, 1st Edition, 1986.

13. Singiresu S Rao. "Mechanical vibrations". Addsion-Wesley, MA, (sixth edition), 2017.

14. Tai-Ran Hsu. "Applied Engineering Analysis: slides for class teaching", Wiley \& Sons, 2018.

15. Wazwaz A.M. "Afirst Course In Integral Equations, Second Edition", World Sci, London, 2015.

16. Wazwaz A.M. "Linear and Non-linear Integral Equations: Method and Applications", springer, London, 2011. 